Cahiers de recherches médiévales

\title{
Du De malo au Malleus Maleficarum
}

Les conséquences de la démonologie thomiste sur le corps de la sorcière

\section{Christine Pigné}

\section{OpenEdition}

Journals

Édition électronique

URL : https://journals.openedition.org/crm/768

DOI : $10.4000 / \mathrm{crm} .768$

ISSN : 1955-2424

Éditeur

Honoré Champion

Édition imprimée

Date de publication : 30 décembre 2006

Pagination : 195-220

ISSN : 1272-9752

Référence électronique

Christine Pigné, «Du De malo au Malleus Maleficarum », Cahiers de recherches médiévales [En ligne], 13। 2006, mis en ligne le 27 novembre 2009, consulté le 15 décembre 2022. URL : http://

journals.openedition.org/crm/768; DOI : https://doi.org/10.4000/crm.768 


\section{RM}

\section{Du De malo au Malleus Maleficarum : les conséquences de la dé- monologie thomiste sur le corps de la sorcière}

Dans son livre intitulé Le Thomisme. Introduction à la philosophie de saint Thomas d'Aquin, Étienne Gilson décrit ainsi la vision thomiste de l'être humain: «En disant que l'âme humaine est 'naturellement unissable' à un corps, on ne signifie pas simplement que, par une rencontre sans fondement dans sa nature, elle peut s'y trouver accidentellement unie ; la sociabilité avec le corps est, au contraire, essentielle à l'âme. " Héritier de l'anthropologie aristotélicienne, saint Thomas s'est détourné du mépris de la chair et a fait preuve d'un véritable «optimisme métaphysique $^{2} »$. Or en 1272, il s'attaque au problème de l'existence du mal et rédige le $D e$ $m_{a l o}{ }^{3}$. Un peu plus de deux siècles plus tard, en 1486, les deux inquisiteurs rhénans, Henry Institoris et Jacques Sprenger, écrivent le Malleus Maleficarum, et décrivent le corps de la sorcière selon les catégories logiques qui s'imposent à eux : celles de la philosophie thomiste ${ }^{4}$. Mandatés pour combattre l'hérésie de la sorcellerie, ces deux auteurs sont le produit de l'institution catholique du $\mathrm{XV}^{\mathrm{e}}$ siècle finissant ${ }^{5}$. Dès l'Apologie, ils soulignent leur mission au sein de l'Église : «Au milieu de ces maux, Nous Inquisiteurs, Jacques Sprenger et son cher collègue, [avons été] délégués par le Siège Apostolique pour exterminer une hérésie aussi pestilentielle. » Cet ouvrage remporte un succès considérable, comme le souligne Amand Danet: "si l'on s'en tient aux conclusions des spécialistes, fixant les éditions des $\mathrm{XV}^{\mathrm{e}}$ et $\mathrm{XVI}^{\mathrm{e}}$ siècles à des chiffres qui oscillent entre mille et quinze cents exemplaires, on peut conclure que plus de trente mille exemplaires du Malleus ont été ainsi mis en circulation à

\footnotetext{
${ }^{1}$ Étienne Gilson, Le Thomisme. Introduction à la philosophie de saint Thomas d'Aquin, Paris, Vrin, 1972, p. 243.

${ }^{2}$ Ibid., p. 227.

${ }^{3}$ Saint Thomas d'Aquin, Questions disputées sur le mal (De malo), traduction par les moines de Fontgombault, Paris, Nouvelles Éditions Latines, 1992. Pour la datation de ce traité, voir A. Boureau, Satan hérétique. Histoire de la démonologie (1280-1330), Paris, Odile Jacob, 2004, note 8 de la p. 128.

${ }^{4}$ Nous citerons toujours le texte du Malleus dans la traduction d'Amand Danet. Voir Henry Institoris et Jacques Sprenger, Le Marteau des sorcières, Malleus Maleficarum, texte traduit du latin et présenté par A. Danet, Grenoble, éd. Jérôme Millon, 2005. Pour la question des deux auteurs, voir l'introduction d'A. Danet, p. 22-33. Nous citerons également en note le texte latin, extrait du Malleus Maleficarum, Lyon, P. Landry, 1595 (nous utiliserons le sigle $M M$ pour désigner cet ouvrage).

${ }^{5}$ Je remercie Jean Céard pour ses relectures et ses précieuses indications concernant les termes latins. Je remercie également Bruno Méniel qui a utilement replacé le projet des deux inquisiteurs dans son contexte historique.

${ }^{6}$ Le Marteau des sorcières, Apologie, éd. cit., p. 101 ; Inter qua mala nos Inquisitores, Iacobus Sprenger, una cum carissimo, ab Apostolica sede, in exterminium tam pestifera Hareseos, socio deputato (MM, Apologia). Voir, à ce propos, «Une peste de sorciers », dans P.-G. Maxwell-Stuart, La sorcellerie et son histoire, éd. Alan Sutton, 2005, p. 65-109.
}

Cahiers de Recherches Médiévales, 13, 2006 
travers l'Europe $»^{7}$. Il est donc légitime de se demander, à la suite d'Alain Boureau, comment «des hommes d'Église, au faîte d'un savoir de plus en plus raffiné et de plus en plus critique ${ }^{8} »$ ont pu utiliser la lumineuse science scolastique pour justifier l'existence des bûchers et répondre ainsi aux craintes de bon nombre de leurs contemporains.

Dans un article intitulé «Le sabbat et la question scolastique de la personne », Alain Boureau étudie l'influence de l'anthropologie aristotélico-thomiste sur la représentation de la sorcière dans le Malleus ${ }^{9}$. La démonologie thomiste rencontre les mêmes interrogations que la philosophie scolastique: «Telle me paraît être la pertinence nouvelle de la thématique démonologique : aux extrêmes de l'expérience, elle pose les questions fondamentales de l'anthropologie scolastique: Qu'est-ce qu'un homme? Quel est le rapport entre l'âme et le corps ? ${ }^{10}$ Selon Alain Boureau, les deux inquisiteurs font une «utilisation assez précise et fidèle de la démonologie de saint Thomas, très attachée à repérer les points de contacts possibles entre Satan et les hommes ${ }^{11} »$. Notre étude se concentrera sur l'influence de la démonologie thomiste sur le corps de la sorcière.

Saint Thomas d'Aquin ouvre son traité de démonologie que constitue la question XVI du De malo, par cette interrogation: «Les démons ont-ils des corps qui leur sont unis naturellement? $\gg^{12}$ Sa réponse commence par cette affirmation : «Le fait que les démons aient des corps qui leur soient naturellement unis, ou qu'ils n'en aient pas, n'a pas beaucoup d'importance pour la doctrine de la foi chrétienne. $»^{13}$ Or, un peu plus de deux siècles plus tard, la logique implacable du Malleus Maleficarum s'articule autour de cette question fondamentale de la corporéité démoniaque. Les deux inquisiteurs rhénans tirent parti de toutes les conclusions de saint Thomas. Le démon thomiste, beaucoup moins puissant que son homologue néoplatonicien, ne peut guère agir dans le monde sensible; il aura donc besoin d'un médium très concret : le corps de la sorcière.

\section{Néoplatonisme et thomisme : la puissance relative du démon}

Le mode de questionnement choisi dans le De malo ${ }^{14}$ permet au lecteur de se faire une idée de la puissance du démon néoplatonicien, contre laquelle saint Thomas s'inscrit en faux. Les auteurs contestés dans le premier article sont tous plus ou moins héritiers du platonisme, comme saint Thomas le rappelle lui-même : «saint

\footnotetext{
${ }^{7}$ Le Marteau des sorcières, introduction, éd. cit., p. 13.

${ }^{8}$ Alain Boureau, "Satan et le dormeur. Une construction de l'inconscient au Moyen Âge », dans Chimères, 14, 1991-1992, p. 41.

${ }^{9}$ A. Boureau, «Le sabbat et la question scolastique de la personne », dans Le sabbat des sorciers $\left(X V^{e}-X V I I I^{e}\right.$ siècles), sous la direction de Nicole Jacques-Chaquin et Maxime Préaud, Grenoble, éd. Jérôme Millon, 1993, p. 33-46.

${ }^{10}$ Ibid., p. 38.

${ }^{11}$ Ibid., p. 34.

${ }^{12}$ De malo, question XVI, article 1, éd. cit., p. 731 : Et primo queritur utrum demones habeant corpora naturaliter sibi unita.

${ }^{13}$ Ibid., p. 738 : Dicendum, quod sive demones habeant corpora naturaliter sibi unita sive non habeant hoc non multum refert ad fidei christiane doctrinam.

${ }_{14}$ Voir, à ce sujet, Marie-Dominique Chenu, Saint Thomas d'Aquin et la théologie, Paris, Seuil, 2005, p. 169-176.
} 
Augustin parle des corps des démons selon ce qu'en ont pensé certains hommes savants, à savoir les Platoniciens ${ }^{15}$ », «Denys [...] suivit en bien des cas la pensée des Platoniciens ${ }^{16} »$. Plotin, Apulée ou Chalcidius sont encore cités ${ }^{17}$. Tous ces auteurs ont en commun d'affirmer l'existence des démons, mais surtout de mettre en valeur une certaine corporéité de ces créatures : intermédiaires entre le divin et l'humain dans le Banquet de Platon $^{18}$, les démons ont des «corps aériens animés ${ }^{19}$ ». À l'inverse, saint Thomas souligne que le Stagirite ne s'est jamais intéressé à de tels intermédiaires : «les Péripatéticiens, disciples d'Aristote, n'ont pas établi qu'il existait des démons, mais ils disaient que ce qui est attribué aux démons provenait de la puissance des corps célestes et des autres réalités naturelles ${ }^{20} »$.

Cet écart originel entre l'intérêt de Platon et le désintérêt d'Aristote pour les démons mérite de retenir quelques instants notre attention. Selon l'élève de Socrate, l'être humain est constitué de deux parties fondamentalement différentes, une âme divine et un corps pesant et mortel ${ }^{21}$. L'âme a partie liée avec le monde des Idées, de l'être en tant qu'être ; le corps, quant à lui, est amené à disparaître. Cette pensée de la rupture n'est pas limitée à la simple anthropologie platonicienne. Le macrocosme et le microcosme sont régis par le même dualisme: le monde sensible s'oppose au monde des Idées. L'homme, composé d'une âme et d'un corps, se tient entre ces deux mondes, dans une situation dramatique ${ }^{22}$. La démonologie inspirée du platonisme cherche très souvent à réconcilier l'homme avec la transcendance ${ }^{23}$. Si les démons pullulent dans le De deo Socratis du pseudo-Apulée par exemple, c'est qu'ils sont nécessaires à la bonne communication entre le monde terrestre et le monde céleste. Les démons et l'imagination jouent d'ailleurs le même rôle de «vecteurs » d'images : les créatures démoniques transmettent les prières et les ordres entre les hommes et les dieux ; l'image et la sensation établissent une communication entre l'âme et le corps. Le démon platonicien permet au Tout d'être véritablement un Tout. Comme l'explique Diotime à Socrate dans Le Banquet, le démon « traduit et transmet aux dieux ce qui vient des hommes, et aux hommes ce qui vient

\footnotetext{
${ }^{15}$ De malo, question XVI, article 1, éd. cit., p. 742 : Augustinus [...] loquitur de corporibus demonum secundum quod visum est quibusdam doctis hominibus, id est Platonicis.

${ }^{16}$ Ibid., p. 742 : Dionisius [...] in plurimis fuit sectator sententie Platonice.

${ }^{17}$ Ibid., p. 734.

${ }^{18}$ Platon, Le Banquet, 202d-203a.

${ }^{19}$ De malo, question XVI, article 1, éd. cit., p. 732 : corpora quedam area animata.

${ }^{20}$ Ibid., p. 739 : Perypathetici Aristotilis sectatores non posuerunt demones esse, set ea que attribuuntur demonibus dicebant provenire ex virtute celestium corporum et aliarum naturalium rerum.

${ }^{21}$ La conception platonicienne du corps a très souvent été analysée. Nous renvoyons à une étude très récente et à la bibliographie donnée en note : Sylvain Roux, «Le statut du corps dans la philosophie platonicienne », dans Le corps, sous la direction de J.-C. Godard, Paris, Vrin, 2005, p. 11-42. François Châtelet étudie également l'évolution de la perception platonicienne de l'intelligible et du sensible dans Platon, Paris, Gallimard, 1965.

${ }^{22}$ Nous reprenons les arguments développés par Jeanne Hersch dans L'étonnement philosophique. Une histoire de la philosophie, Paris, Gallimard, 1993, p. 54.

${ }_{23}$ Pour une étude détaillée de la démonologie platonicienne et néoplatonicienne, voir l'introduction de J. Beaujeu, dans Apulée, Opuscules Philosophiques et Fragments, texte établi et trad. par J. Beaujeu, Paris, Belles Lettres, 1973, p. VII-XLIX.
} 
des dieux : d'un côté les prières et les sacrifices, de l'autre les ordres et la rétribution des sacrifices, et comme il est à mi-chemin des uns et des autres, il contribue à remplir l'intervalle, de manière que le Tout soit lié à lui-même ${ }^{24}$ ». Les démons permettent de jeter un pont entre deux mondes que tout oppose, celui de la matière et celui des Idées.

Selon Aristote au contraire, l'âme et le corps humain ne sont pas entièrement dissociables. L'âme est la forme du corps, son entéléchie : "Nommée phusis, nature, lorsqu'il s'agit de réalités plus simples, la puissance active de la forme est l'âme de l'être vivant [...] Cette définition signifie que l'âme est la forme parfaite et immuable, en tant que détermination achevée du type spécifique, et la puissance active adaptée à la puissance passive de l'organisme qui lui correspond : comme telle, l'âme est la faculté vitale qui peut entrer en plein exercice en «animant» son corps, dont elle dirige, en effet, toutes les fonctions afin de s'en servir comme d'un instrument. $»^{25}$ On comprend désormais pourquoi la démonologie n'intéresse guère le Stagirite. Aux deux niveaux platoniciens de l'être, les choses sensibles et le monde des Idées, Aristote oppose une échelle des êtres : "la matière inerte ; la matière organique; l'organisme vivant; dans l'organisme vivant, les organes. Grâce à la forme, les organes constituent l'unité d'un être vivant; et c'est cette forme qu'Aristote appelle l'âme. Ce terme n'est pas pris dans son sens spirituel humain : il désigne le principe de vie qui crée l'unité du corps vivant ${ }^{26} »$. Selon Aristote, l'être humain présente une belle unité, à l'image même de celle du cosmos. Point n'est besoin d'un quelconque démon pour rapprocher le monde terrestre et le monde divin.

$\mathrm{Au} \mathrm{XIII}^{\mathrm{e}}$ siècle, saint Thomas tente de réaliser une synthèse entre l'aristotélisme et la tradition chrétienne ${ }^{27}$. À la suite d'Aristote, il cherche à «mettre toujours en évidence la continuité. Le sens du tragique, de la déchirure, de la rupture lui est étranger ${ }^{28}{ }$. Dans l'échelle des êtres qui montent vers Dieu, Thomas d'Aquin rajoute la classe des anges : l'âme humaine est la plus haute des formes matérielles ; elle est aussi la plus basse des formes pures. "Au dessus d'elle s'élève la hiérarchie des formes pures, sans aucune matière : c'est la hiérarchie des anges. ${ }^{29}$ Or en 1272, saint Thomas se montre soucieux d'intégrer les démons - si importants dans le

\footnotetext{
${ }^{24}$ Platon, Le Banquet, 202 e, CEuvres complètes, tome IV, deuxième partie, trad. de P. Vicaire, notice de L. Robin, Paris, Les Belles Lettres, 1992.

${ }^{25}$ Jean Bernhardt, dans Histoire de la Philosophie, tome I, « La philosophie païenne. Du VIe siècle av. J.-C. au III ${ }^{\mathrm{e}}$ siècle ap. J.-C. », sous la direction de François Châtelet, Hachette Littérature 1999, p. 174. Voir également J. Hersch, L'étonnement philosophique, éd. cit., p. 62. La conception aristotélicienne du corps a très souvent été analysée. Nous renvoyons à une étude très récente et à la bibliographie donnée en note: Marie-Hélène Gauthier-Muzellec, «La matière du corps vivant chez Aristote », dans Le corps, éd. cit., p. 43-84.

${ }^{26} \mathrm{~J}$. Hersch, L'étonnement philosophique, éd. cit., p. 63.

${ }^{27}$ Voir É. Gilson, Le Thomisme, éd. cit. Voir également The Cambridge History of Later Medieval Philosophy, N. Kretzmann, A. Kenny et J. Pinborg ed., Cambridge University Press, 1982.

${ }^{28}$ J. Hersch, L'étonnement philosophique, éd. cit., p. 115.

${ }^{29}$ Ibid., p. 114.
} 
christianisme ${ }^{30}-$ dans l'univers qu'il explore avec passion depuis si longtemps. Comme nous l'avons vu, il se souvient du désintérêt aristotélicien pour les créatures démoniques. Cette tension entre l'héritage chrétien et le modèle aristotélicien explique en partie la naissance du démon thomiste: l'auteur du De malo admet l'existence des démons, mais leur supprime tout corps. Comme le note Alain Boureau, les démons thomistes sont peu puissants : «En somme, les démons de Thomas d'Aquin n'ont guère de vivacité. Il ne s'agit sans doute pas là d'une simple limite de l'explication, mais d'une orientation théologique en direction d'un christianisme de la lumière pour qui le Christ a contenu et enchaîné les démons, qui ne bénéficieront d'une liberté brève que dans le court moment précédant le jugement dernier, hors de quelques évasions fermement contrôlées. L'essentiel de leur histoire s'est produit au second instant de la création. Depuis, ils gèrent tristement leur médiocre capacité de nuisance, marginale et maîtrisable. ${ }^{31}$ Les démons thomistes n'ont pas de corps. Pour agir concrètement dans le monde sensible, ils auront donc besoin d'un agent intermédiaire. Privé de tout support corporel, le démon thomiste ne peut pas véhiculer des images dans l'esprit des hommes : la démonologie du De malo n'a donc plus rien à voir avec la démonologie néoplatonicienne, si soucieuse de mettre en rapport l'imagination humaine et les corps démoniques. Deux siècles plus tard, les deux auteurs du Malleus développent consciencieusement toutes les conséquences de telles affirmations pour mieux définir ce qui les intéresse : la sorcière.

leus

Le démon incorporel et le corps de la sorcière : du De malo au Mal-

Le démon néoplatonicien est une créature puissante. Muni d'un corps, il peut tout à fait exercer ses nuisances directement sur sa victime. Saint Thomas - qui s'apprête à récuser une telle affirmation - présente toutefois les arguments de ses adversaires : «De plus, rien ne peut agir sur une chose à distance, à moins que sa force ne soit transmise par un intermédiaire. Or la puissance d'un esprit pur ne peut être transmise par un intermédiaire corporel, parce qu'un corps ne peut recevoir une force spirituelle. Donc, puisque le démon agit sur des choses à distance, il semble qu'il ne soit pas un esprit pur, mais un être composé de corps et d'esprit. $»^{32}$ Dans la réponse que saint Thomas apporte aux objections platoniciennes, ce dernier argument est énergiquement réfuté :

Il reste donc que le corps serait uni à l'âme principalement en vue de la sensation, comme cela se produit aussi pour nous. Aussi les Platoniciens ont-ils admis que les

\footnotetext{
${ }^{30}$ Voir Norman Cohn, Démonolâtrie et sorcellerie au Moyen Âge. Fantasmes et réalités, Paris, Payot, 1982.

${ }^{31}$ Alain Boureau, Satan hérétique, éd. cit., p. 141.

${ }^{32}$ De malo, question XVI, article 1, éd. cit., p. 735-736: Preterea. Nichil potest agere in aliquid distans nisi virtus eius per medium deferatur in illud. Virtus autem spiritus puri non potest deferri per medium corporale, quia corpus non est capax spiritualis virtutis. Cum ergo demon agat in aliquid distans, videtur quod non sit spiritus purus set sit aliquid compositum ex corpore et spiritu.
} 
démons étaient des êtres animés à l'âme passible ${ }^{33}$; ce qui relève de la partie sensible. Mais la sensation ne peut exister sans le toucher, qui est le fondement de tous les autres sens ; aussi lorsqu'il se corrompt, l'animal se corrompt aussi ; or l'organe du toucher ne peut être un corps aérien, ni quelque corps simple, comme on le prouve au livre de l'Ame (III, 11). Aussi reste-t-il que nul corps aérien ne peut être animé : et c'est pourquoi nous disons que les démons n'ont pas de corps qui leur soient naturellement unis ${ }^{34}$.

L'article 9 de la question XVI du De malo tire des conséquences pratiques de cette définition ontologique des démons. S'inscrivant parfaitement dans un cadre de pensée aristotélicien, saint Thomas souligne la distance qui sépare les substances immatérielles que sont les démons et les corps matériels : «les substances immatérielles ne peuvent pas transformer la matière quant à la forme. Or les démons sont des substances immatérielles. Donc il semble qu'ils ne puissent pas transformer formellement les corps matériels. $»^{35}$ Saint Thomas propose donc l'intervention de «certains agents corporels ${ }^{36} »$, servant de liaisons, d'intermédiaires, entre la volonté perverse du démon et sa future victime : «Tout ce qui s'accomplit visiblement en ce monde peut être fait par les démons non par leur seule puissance propre, mais en utilisant des agents naturels, comme on l'a dit. Le démon se sert comme d'un instrument d'un agent naturel pour produire un certain effet. ${ }^{37}$ Dans cet article 9, saint Thomas évoque une seule fois le rôle des «magiciens» qui contractent des «pactes privés avec les démons ${ }^{38} »$. Deux siècles plus tard, Henry Institoris et Jacques Sprenger n'auront guère de mal à faire de la sorcière cet «instrument» des démons, capable de concrétiser dans le monde sensible les volontés perverses des créatures infernales.

\footnotetext{
${ }^{33}$ Nous traduisons animalia animo passiva par «êtres animés à l'âme passible», plutôt que «êtres animés à l'esprit passif », comme le proposent les moines de Fontgombault. Les néoplatoniciens distinguent en effet l'animus et la mens. En traduisant par «âme», nous évitons de mettre ainsi la pars sensitiva dans l'esprit. D'autre part, par passivus, les néoplatoniciens entendent la capacité d'éprouver de la colère, de la pitié, etc., ce que rend mal le terme «passif ». L'adjectif «passible» semble donc plus indiqué.

${ }^{34}$ Ibid., p. 741-742 : relinquitur ergo quod hoc sit principaliter propter sensum, sicut et in nobis accidit. Unde et Platonici posuerunt demones esse animalia animo passiva; quod pertinet ad partem sensitivam. Sensus autem non potest esse sine tactu qui est fundamentum omnium sensuum, unde eo corrupto corrumpitur animal; organum autem tactus non potest esse corpus aereum nec aliquod corpus simplex, ut probatur in libro De anima. Unde relinquitur quod nullum corpus aereum possit esse animatum: et ideo dicimus demones non habere corpora naturaliter sibi unita.

${ }^{35}$ Ibid., article 9, p. 849-850: substantie immateriales non possunt transmutare materiam ad formam. Set demones sunt substantie immateriales. Ergo videtur quod non possunt formaliter corpora materialia transmutare.

${ }^{36}$ Ibid., p. 851 : aliqua corporalia activa.

${ }^{37}$ Ibid., p. 851: Ad primum ergo dicendum quod omnia que visibiliter hunt in hoc mundo, possunt heri per demones non sola propria virtute, set mediantibus activis naturalibus, ut dictum est. Ad secundum dicendum quod demon utitur activo naturali ad aliquem effectum producendum sicut instrumento.

${ }^{38}$ Ibid., p. 854 : magi [...] per privatos contractus demonum.
} 
Les deux auteurs du Malleus Maleficarum reprennent scrupuleusement les arguments aristotéliciens maniés par saint Thomas : «Enfin entre agent et patient, il doit y avoir proportion. Mais il ne peut y avoir proportion entre substance purement spirituelle et substance corporelle. D'où les démons eux-mêmes ne peuvent rien pour un effet corporel sinon par l'intermédiaire d'un autre principe actif. $»^{39}$ L'instrumentum thomiste devient donc très naturellement la sorcière: «Notre opinion toutefois reste que le diable ne peut rien faire ici bas sans sorciers. Et d'abord dans l'ordre de la génération, où toute action se fait par contact. Puisqu'il n'y a point contact direct du démon avec le corps - rien de commun entre eux - il doit donc se servir d'un instrument à qui il donne de faire le mal par contact. ${ }^{40}$ Le démon thomiste, privé de corps, a besoin de la sorcière pour répandre ses méfaits sur les humains. Il semble impossible de s'en prendre à une substance immatérielle perverse telle que la volonté démoniaque, alors qu'il est tout à fait possible de brûler concrètement le corps de la sorcière. Tuer ces agents de Satan revient à affaiblir la puissance démoniaque. Faire mourir les sorcières, c'est priver le démon de tout support corporel, c'est le priver de toute possibilité d'action sur le monde physique. La sorcière est un médium très concret entre le démon et la victime, qu'il est possible d'éradiquer une bonne fois pour toutes. Encore faut-il reconnaître la réalité de ce médium :

Ces faux interprètes sont encore plus répréhensibles, quand ils concèdent que les extrêmes sont réels (le démon d'un bout et la maladie de l'autre), mais que le médium (instrumental), la personne maléfique, n'intervient qu'en imagination. Le médium en effet participe toujours de la nature des extrêmes. Et il ne sert à rien de dire que l'imagination c'est du réel : l'imagination comme telle ne peut agir ni participer à l'action du démon que sur la base d'un pacte où la sorcière s'est offerte et s'est liée tout entière au démon, vraiment et réellement, et non seulement en fantaisie et en imagination. Il faut donc reconnaître aussi qu'elle collabore avec le diable réellement et corporellement. C'est d'ailleurs à cela que tendent les éléments du maléfice : les sorciers opérant toujours soit par un regard soit par une formule magique déposée sous le seuil de la maison, comme on verra à la question suivante ${ }^{41}$.

\footnotetext{
${ }^{39}$ Le Marteau des sorcières, Première partie, quest. III, éd. cit., p. 135 ; Demum quia inter agens et patiens debet esse proportio, nulla potest esse proportio inter substantiam pure spiritalem et corporalem : ideo nec ipsi Damones possent in aliquem effectum, nisi mediante aliquo alio principio activo (MM, p. 35).

${ }^{40}$ Ibid., quest. II, p. 117 ; Sed contra quod nihil possit inficere inferior absque maleficis. Primo de generatione, omnis actio est per contactum. Et quia non est aliquis contactus damonis ad corpora, cum nihil habeat cum eis commune, ergo utitur aliquo instrumento illi influendo virtutem laedendi per contactum (MM, p. 14). Voir également Le Marteau des sorcières, éd. cit., p. 110 , p. 119 , p. 127 , p. 135 , p. 154, p. 168, p. 248 . Voir par exemple dans le De malo : «De plus, toute action se fait par contact, comme on le dit dans la Génération (I, 6). Or il ne semble pas qu'il puisse y avoir de contact entre le démon et les corps, parce qu'il n'a avec eux rien de commun »: Preterea. Omnis actio est per contactum, ut dicitur in I De generatione. Non videtur autem quod possit esse aliquis contactus demonis ad corpora: quia nichil habet cum eis commune (éd. cit., question XVI, article 9, p. 855).

${ }^{41}$ Le Marteau des sorcières, Première partie, quest. I, éd. cit., p. 111 ; Praterea tales sic errantes peramplius reprehenduntur dum extrema concedunt realia, scilicet damonem oper-
} 
Les deux inquisiteurs suggèrent même que le démon, capable de manipuler seul l'imagination de sa victime, comme nous le verrons dans une partie suivante, préfère la plupart du temps agir par l'intermédiaire de la sorcière : «il préfère infiniment plus blesser [les hommes] par les sorcières. D'abord parce qu'il porte à Dieu un soufflet plus humiliant en s'appropriant une créature qui lui est consacrée; deuxièmement, l'offense de Dieu étant plus grande, (Dieu) lui laisse davantage de pouvoir de nuire aux hommes ; troisièmement enfin, il y trouve davantage son bénéfice, qu'il place dans la perdition des âmes. ${ }^{42}$ Le démon, seul, est peu puissant ; mais l'alliance perverse du démon et de la sorcière est d'une redoutable efficacité : «En conclusion, ce que le démon peut par lui-même, il le peut davantage encore par les sorcières : ils peuvent nuire aux hommes en tout sans exception. ${ }^{43}$ La mise à mort de la sorcière est donc nécessaire ${ }^{44}$. S'étant donnés corps et âme au démon, ces suppôts de Satan encourent la peine capitale : «La loi divine en effet à plusieurs reprises prescrit non seulement de fuir les sorcières mais de les mettre à mort. On n'imposerait pas semblables peines si ces femmes ne concouraient pas réellement avec les démons pour des dommages et des atteintes réelles et effectives. La mort corporelle n'est infligée que pour un péché grave et physique, à la différence de la mort de l'âme qui peut venir d'une illusion ou d'une autre tentation simplement imaginaire. ${ }^{45}$ Une anecdote rapportée dans la première partie du Malleus est en ce sens très révélatrice. L'un des deux inquisiteurs trouva un jour une ville ravagée par la peste. Le bruit courait qu'une femme morte et enterrée mangeait petit à petit le linceul dans lequel elle avait été ensevelie. L'épidémie était liée à cette terrible ingestion. «On tint conseil à ce sujet. Prévôt et maire de la ville creusant la tombe,

antem, et effectum morbi realem, medium autem instrumenti, scilicet personam maleficam, dicunt fantastice concurrere, cum tamen medium semper naturam participat extremorum. Nec valet si dicatur quod etiam phantasia sit quid reale, quia sicut phantasia ut talis, nil potest efficere nec concurrere ad damonis operationem nisi per pactum initum cum damone in quo pacto malefica se totam obtulit et astrinxit, diabolo vere et realiter et non phantastice aut imaginarie solum : ita etiam oportet quod cooperetur diabolo vere corporaliter. Nam et ad hoc sunt omnia maleficiorum opera, ubi semper aut per visum aut per locutionem alicuius maleficii instrumenti repositi sub limine domus operatione sua maleficia exercent, prout in sequenti quastione patebit (MM, p. 7-8).

${ }^{42}$ Ibid., Deuxième partie, Première quest. principale, chap. VII, p. 289 ; In infinitum enim plus affectat per Maleficas ladere. Tum quia maiorem infert Deo contumeliam, creaturam sibi dicatam usurpando. Secundo, quia cum Deus amplius offenditur, amplius sibi nocendi hominibus potestas permittitur. Tertio propter sui lucrum, quod in perditionem statuit animarum (MM, p. 200).

${ }^{43}$ Ibid., chapitre XI, p. 309 ; Quare concluditur, quod sicut per se, ita et amplius per Maleficas, in omnibus nihil dempto hominibus nocere possunt (MM, p. 222). Voir également Le Marteau des sorcières, éd. cit., p. 298, p. 308 et p. 327.

${ }^{44}$ Pour une description juridique des différentes peines encourues par les sorcières, voir Ibid., Troisième partie, p. 399-524.

${ }^{45}$ Ibid., Première partie, quest. I, p. 107 ; Nam lex divina in plerisque locis, pracipit maleficas non solum esse vitandas, sed etiam occidendas, cuiusmodi ponas non imponeret si non veraciter et ad reales effectus et lasiones cum damonibus concurrerent. Mors enim corporaliter non infigitur sine corporali et gravi peccato, sed aliud de morte anima qua ex phantastica illusione seu etiam tentatione oriri potest (MM, p. 4). 
trouvèrent presque la moitié du linceul engagée dans la bouche, la gorge et l'estomac et déjà digérée (pourrie). Devant ce spectacle, le prévôt bouleversé tira son épée et coupant la tête, la jeta hors de la fosse. Aussitôt la peste cessa. $»^{46}$ Le corps de la sorcière est une menace pour l'humanité, même à l'état de cadavre. La dépouille de la vieille femme reste un médium encore actif entre les volontés démoniaques et le monde des humains. Cet intermédiaire corporel doit être mis à mort deux fois pour que cessent ses terribles maléfices.

La délicate question du sabbat des sorcières : $d u$ De malo au Malleus

Les auteurs du Malleus tirent parti de ce qui n'était qu'esquissé dans le De malo. La nécessité du corps sorcier est justifiée philosophiquement et théologiquement par des arguments aristotélico-thomistes très rigoureux. Il en va de même pour le déplacement du corps de la sorcière lors du sabbat ${ }^{47}$ : ce que saint Thomas ne fait que suggérer, est repris et systématiquement amplifié par les deux inquisiteurs. À l'interrogation qui ouvre l'article 10 de la question XVI du De malo, «Les démons peuvent-ils déplacer les corps ${ }^{48}$ ?», l'auteur répond par l'affirmative : «De même donc que les substances spirituelles supérieures meuvent les corps célestes supérieurs, de même aussi les inférieures, tels les démons, peuvent mouvoir localement les corps inférieurs. ${ }^{49}$

Henry Institoris et Jacques Sprenger reprennent cet argument thomiste, mais lui donnent une portée qu'il était loin d'avoir dans le De malo (l'article 10 étant l'un des plus courts de la question XVI). Les deux inquisiteurs affirment que la sorcière se déplace réellement lors du sabbat, aidée en cela par le démon. Ils s'opposent ainsi à certains lecteurs du Canon Episcopi qui ne parlent que d'un «simple transport fantasmatique, alors que parfois il y a transport réel et corporel ${ }^{50} »$. Une telle croyance finirait par excuser les sorcières et se révèlerait alors extrêmement dangereuse : «Comme si leurs actes portant atteinte aux hommes, aux animaux, aux fruits de la terre, ne devaient pas leur être attribués : leurs transports étant purement fantasmatiques, de même elles se feraient illusion sur les torts qu'elles causent. ${ }^{51}$ Le

${ }^{46}$ Ibid., quest. XV, p. 216 ; Habito consilio desuper, Scultetus cum prafecto civitatis sepulchrum effodientes, invenerunt quasi dimidietatem linteaminis per os et collum usque in ventrem immissam et consumptam. Quo vivo, motus Scultetus, gladium extraxit, et caput amputando extra foveam proiecit, sicque subito pestis cessavit (MM, p. 125).

${ }^{47}$ Voir, à ce sujet, A. Boureau, «Le sabbat et la question scolastique de la personne », art. cit., p. 36.

${ }^{48}$ De malo, question XVI, article 10, éd. cit., p. 855 : Decimo queritur utrum demones possent corpora movere localiter.

${ }^{49}$ Ibid., article 9, p. 858 : Sicut ergo superiores substantie spirituales movent superiora celestia corpora, ita etiam inferiores movere possunt localiter inferiora corpora quales sunt demones.

${ }^{50}$ Le Marteau des sorcières, Deuxième partie, Première quest. principale, chap. II, éd. cit., p. 255 ; solummodo imaginarie, cum tamen interdum etiam vere et corporaliter transferantur (MM, p. 162).

${ }^{51}$ Ibid., chap. III, p. 262 ; tanquam ut et singula earum facta in lasionibus hominum, iumentorum, terra frugum, non sint eis applicanda, eoque sicut phantastice in transvectionibus, ita et circa creaturarum lassiones illudantur (MM, p. 170). 
chapitre intitulé «Comment les sorcières se transportent d'un endroit à un autre ? "2 $^{52}$ est entièrement consacré au déplacement des sorcières lors du sabbat. Les deux inquisiteurs empruntent la majorité de leurs arguments au De malo, mais se montrent beaucoup plus précis dans leur démonstration :

Premièrement la puissance spirituelle, donc aussi la puissance de l'ange ou même de l'âme est plus forte que la puissance corporelle. Deuxièmement (quant à l'âme) : toute forme corporelle est individuelle de par la matière et déterminée dans le temps et dans l'espace en tant qu'elle existe comme notre âme ; les formes immatérielles par contre sont des intelligences absolues et donc ont une puissance absolue et plus universelle. C'est pourquoi l'âme unie au corps ne peut subitement le transporter d'un endroit dans un autre ni l'élever dans les airs. Elle le pourrait si elle était séparée et si Dieu le permettait ; mais tout cela à plus forte raison est possible à l'esprit totalement immatériel, comme est l'ange bon ou mauvais. [...] Donc on doit dire que, ni en tant que corps ni en raison de son âme, le corps humain ne peut faire obstacle à ce qu'il soit transporté subitement, Dieu le permettant, d'un endroit dans l'autre par une substance spirituelle $[\ldots]^{53}$.

Cette dernière phrase prouve bien que, lors du déplacement sabbatique, la sorcière reste une entité âme-corps (particulièrement verrouillée par l'anthropologie aristotélico-thomiste), soulevée dans les airs par la puissance entièrement spirituelle et totalement immatérielle qu'est le démon. La comparaison développée dans l'article 10 de la question XVI du De malo semble pourtant travailler souterrainement l'imaginaire des deux inquisiteurs. Saint Thomas avait établi un parallèle entre l'âme et le corps d'un être humain et le démon et le corps qu'il véhicule : «L'âme humaine occupe le plus bas degré dans l'ordre des substances spirituelles, aussi elle n'a pas le pouvoir de mouvoir un corps, même localement, à moins qu'il ne lui soit proportionné en étant vivifié par elle. Entre le démon et le corps, il n'y a pas contact corporel mais virtuel, qui réclame cependant une convenance de proportion entre le moteur et le mobile. ${ }^{54}$ Dans le Malleus, le démon est le «moteur » de la sorcière,

${ }^{52}$ Ibid., p. 261-268 ; De modo, quo localiter transferuntur de loco ad locum (MM, p. 169177).

${ }^{53}$ Ibid., p. 265-266 ; Primo, quia virtus spiritualis fortior virtute corporali, sicut virtus Angeli, aut etiam anima superior quam virtus corporalis. Secundo quo ad animam : Quia cum omnis forma corporalis est forma individua per materiam, et determinata per hac, et nunc prout iam anima nostra existit, forma autem immateriales sunt absoluta et intelligibiles. Unde etiam potestatem habent absolutam et universaliorem. Ideo anima coniuncta non potest ita subito transferre corpus suum localiter, nec in altum elevare. Bene autem faceret, cum esset separata, Deo permittente : qua omnia a fortiori potest spiritus omnino immaterialis : prout est Angelus tam bonus quam malus. [...] Ideo dicere oportet, quod ratio corporis humani, nec quantum ad ipsum corpus, nec quantum ad ipsam animam obstare potest, quin utrunque moveri possit subito, Deo permittente, de loco ad locum a spirituali substantia (MM, p. 173174).

${ }^{54}$ De malo, question XVI, article 9, éd. cit., p. 859: Ad secundum dicendum quod anima humana infimum gradum tenet in ordine substantiarum spiritualium, unde non habet virtutem movendi corpus, etiam localiter, nisi proportionatum sibi per hoc quod est vivificatum ab ea. Ad tertium dicendum quod demonis ad corpus non est contactus corporalis set virtualis, qui quidem requirit convenientiam proportionis moventis ad mobile. 
qui est son «mobile». La substance spirituelle qu'est la créature démoniaque s'empare de l'âme et du corps de la sorcière, mais devient aussi, d'une certaine façon, l'âme qui informe et vivifie la sorcière, assimilée à un simple corps.

Une telle affirmation ne se donne jamais à lire explicitement sous la plume des deux inquisiteurs et pour cause ! Le Malleus ne cesse de clamer la responsabilité pleine et entière de la sorcière, qui s'est donnée corps et âme au démon: "mais outre le reniement, c'est aux démons eux-mêmes qu'elles se livrent, offrant l'hommage de leurs corps et de leurs âmes ${ }^{55}$ »; "le démon réclame 'l'hommage' : qu'elle se donne à lui corps et âme pour toujours ${ }^{56} »$. La sorcière reste avant tout un être humain: c'est ce qui rend sa conduite d'autant plus scandaleuse. Soulignant l'alliance nécessaire de la sorcière et du démon, les deux inquisiteurs évacuent de ce fait d'autres causes avancées pour expliquer les phénomènes occultes : le destin, la fortune, l'astrologie, etc. ${ }^{57}$. Réfutant l'influence des astres sur les jeteurs de sorts, les deux auteurs du Malleus soulignent la «normalité » de la sorcière, et empruntent leur argument principal à la psychologie aristotélicienne :

Deuxièmement (les œuvres des sorciers) ne proviennent pas non plus de paroles prononcées par n'importe quels hommes sur des images quelconques, sous une quelconque constellation. L'intelligence de l'homme en effet est disposée de manière à connaître à partir des choses : pour comprendre nous avons besoin de partir des fantasmes. Il n'est pas dans notre nature, simplement par un concept ou une opération interne de l'esprit, de causer des choses extérieures ou de changer les choses, simplement en prononçant des mots. Des hommes qui auraient pareil pouvoir ne seraient pas de la même nature que nous : leur appliquer à eux le mot «homme » serait équivoque ${ }^{58}$.

La sorcière s'est donnée corps et âme au démon : elle mérite entièrement son sort, puisque sa responsabilité morale et spirituelle est engagée dans ses actes ${ }^{59}$. Tout

\footnotetext{
${ }^{55}$ Le Marteau des sorcières, Première partie, question XIV, éd. cit., p. 215 ; Sed ipsis Damonibus ultra abnegationem, homagium, corpora et animas offerendo prestant (MM, p. 124).

${ }^{56}$ Ibid., Deuxième partie, Première quest. principale, chap. II, éd. cit., p. 254 ; Damon homagium petit, quod continet, ut in anima et corpore, sibi aternaliter pertineat (MM, p. 162).

${ }_{57}$ Voir essentiellement à ce sujet, Ibid., Première partie, question V, éd. cit. : «D'où vient la prolifération des œuvres des sorcières? », p. 142-157; Unde multiplicatio maleficorum operum procedat (MM, p. 43-60)

${ }^{58}$ Ibid., p. 153 ; Demum quod nec ex vocibus quorumcunque hominum, concurrente quacunque constellatione, super quascunque imagines. Nam cum intellectus hominis huius dispositionis est, quod eius cognitio ex rebus causatur : cum intelligentem necesse sit Phantasmata speculari : non est eius conditio, quod ex sua conceptione seu intellectuali operatione intrinseca, ubi illam solam per verba exprimeret, res ab extra habeat causare : aut quod conceptio intellectus expressa per verba haberent corpora immutare. Tales enim homines qui talem haberent virtutem, non essent nobiscum unius speciei, sed aequivoce dicerentur homines (MM, p. 56).

${ }_{59}$ «Si l'on se fie au Malleus, les sorciers (ou plus exactement les auteurs de maléfices, les malefici) ont toujours existé ; mais la nouveauté, depuis l'an 1400, c'est que ces malefici ne sont plus seulement des victimes du diable, mais engagent leur volonté. Certes, cette affirmation ne convainc guère puisque, on l'a dit, le thème du pacte satanique paraît fort ancien. Mais le point de vue des auteurs importe, puisqu'il s'applique à la région la plus sensible de
} 
se passe pourtant comme si les deux inquisiteurs étaient terriblement gênés par «l'âme » de la sorcière - chasse gardée de Dieu - et cherchaient par tous les moyens à s'en débarrasser. Comment concilier en effet la présence divine de Dieu dans l'âme («il n'est pas possible au démon d'habiter dans l'âme puisque Dieu seul habite l'esprit $\left.{ }^{60} »\right)$ et la perversion de l'esprit de la sorcière («offrir son patronage à ceux qui agissent contre la vertu n'est pas la marque d'un esprit bien disposé61 ») ? Une lecture attentive du chapitre intitulé «Comment se fait la profession sacrilège et comment se rend l'hommage ? ${{ }^{62}}^{2}$ peut apporter une réponse à cette question essentielle. Comparons deux passages consacrés respectivement à la sorcière et au démon et séparés de quelques pages l'un de l'autre ${ }^{63}$ :

- les sorcières :

Ce sont celles-là qui, allant contre l'inclination de la nature de l'homme et même des animaux, ont coutume de dépecer et de dévorer (vorant, et comedere solent) les enfants de leur propre espèce. C'est la plus importante espèce en sorcellerie. Elles visent à d'innombrables autres maux. En effet elles soulèvent (exagitant) les tempêtes et des orages avec des éclairs; elles provoquent la stérilité (sterilitatem procurant) chez les hommes et chez les bêtes. Les enfants qu'elles ne dévorent pas (devorant), elles les offrent (offerunt) aux démons ou elles les tuent (occidunt) autrement: ce sont ceux qui ne sont pas renés de la fontaine du baptême. Ceux qu'elles dévorent (devorant) (non sans la permission de Dieu), ce sont des baptisés. Des enfants marchant au bord de l'eau, elles savent sans se faire voir les jeter (proiicere) dans l'eau en présence même des parents. Elles peuvent rendre les chevaux fous (freneticos facere) sous leurs cavaliers; elles peuvent se transporter (transmeare) d'un endroit dans l'autre à travers les airs, soit en corps soit en esprit; elles peuvent changer ( $\mathrm{va}$ leant immutare) le cœur des juges et des magistrats de manière à ce que qu'ils ne puissent leur nuire. Dans les tortures elles peuvent se doter elles-mêmes et doter les autres de «taciturnité » (sibi et aliis procurare) ; déclencher un tremblement (tremorem magnum incutere) dans les mains et les âmes de ceux qui viennent les arrêter; révéler (manifestare) aux autres des choses occultes et des événements futurs, au moins, selon saint Thomas, ceux qui peuvent avoir une cause naturelle et que le démon peut connaître. Elles peuvent voir (conspicere) des choses absentes comme si elles étaient présentes ; changer (immutare) les cœurs des hommes pour un amour ou une haine désordonnés; détruire (interimere) parfois ce qu'elles veulent par la foudre, même des hommes et des bêtes. Elles peuvent rendre stérile la puissance génitale ou même rendre impossible l'union conjugale (vim generativam, aut etiam

l'anthropologie scolastique : celle de la volonté et du libre-arbitre » (A. Boureau, «Le sabbat et la question scolastique de la personne», art. cit., p. 35).

${ }^{60}$ Le Marteau des sorcières, Deuxième partie, Première quest. principale, chap. $\mathrm{X}$, éd. cit., p. 300 ; non est Diabolo possibile habitare in anima, eo quod solus Deus menti illabitur (MM, p. 212).

${ }^{61}$ Ibid., Première partie, question V, p. 150 ; Nam prastare patrocinium aliquibus in his, quae fiunt contraria virtuti, non est alicuius intellectus bene dispositi (MM, p. 52).

${ }_{62}$ Ibid., Deuxième partie, Première quest. principale, chap. II, p. 253-261; Sequitur de modo sacrilega professionis (MM, p. 160-169).

${ }^{63}$ Les termes latins importants sont indiqués entre parenthèses. 
potentia coeundi auferre), provoquer (procurare) l'avortement, causer la mort (interimere) des enfants dans le sein de leur mère rien que par un attouchement extérieur. Elles peuvent parfois ensorceler (maleficiare) des hommes ou des animaux par un simple regard, sans le moindre toucher, et ainsi causer la mort (mortem inferre), vouer (dedicare) leurs propres enfants aux démons ${ }^{64}$.

- les démons :

selon Augustin il y a sept causes à partir desquelles ils peuvent conjecturer (conjecturant) avec probabilité des futurs contingents sans qu'ils puissent (valeant) le savoir (scire) avec certitude. La première, c'est qu'ils jouissent d'une subtilité naturelle quant à l'opération intellectuelle (ad operationem intellectus ipsorum); d'où ils comprennent (intelligunt) sans le raisonnement qui nous est nécessaire. La deuxième, c'est que, par expérience des temps et révélation d'esprits supérieurs, ils savent (sciunt) davantage de choses que nous. D'où Isidore dit (ce que les Docteurs ont souvent cité) que les démons disposent d'une triple acuité intellectuelle (triplici acumine scire vigent) : la subtilité de nature, l'expérience des temps et la révélation des esprits supérieurs. La troisième cause, c'est la rapidité du mouvement par laquelle avec une vitesse étonnante ils peuvent anticiper (pracedere) en Occident des choses encore à faire en Orient. La quatrième cause, c'est que, de même qu'ils sont capables avec la permission de Dieu de déclencher des maladies, d'empoisonner l'air et de provoquer la famine, de même ils peuvent prédire (predicere) tout cela. La cinquième cause, c'est qu'ils peuvent plus habilement prédire (predicere) la mort à partir de signes que le médecin ne peut le faire en examinant l'urine ou en tâtant le pouls. Car comme le médecin remarque dans un malade des signes que le profane ne remarque pas, ainsi le démon voit (videt) naturellement ce que nul homme ne voit. La sixième cause, c'est qu'il peut, à partir des signes procédant de l'âme d'un homme, conjecturer (conjecturant) ce qu'il y a ou ce qu'il y aura en son cœur plus finement qu'un homme prudent. Ils savent en effet quelles impulsions et donc quelles actions vont s'ensuivre. La septième cause, c'est qu'ils connaissent (noscunt) mieux que les hommes les actes et les écrits des prophètes; d'où, puisque beaucoup de choses futures en dépendent, ils peuvent à partir de là en prévoir (prcedicere) beaucoup ${ }^{65}$.

Dans ces deux passages très proches l'un de l'autre, les deux inquisiteurs décrivent - non sans une certaine fascination - les terribles pouvoirs de la sorcière et l'effrayante puissance des démons. Les deux extraits semblent construits suivant la même logique : le verbe «valere » en latin, qui connote la puissance et la force, martèle presque toutes les phrases. La puissance de la sorcière et celle des créatures démoniaques n'ont pourtant rien à voir. Les verbes utilisés pour illustrer les «maux » de la sorcière sont des verbes d'action et dépendent presque tous d'un imaginaire corporel. Au contraire, les verbes qui traduisent la puissance démoniaque appartiennent tous au vocabulaire de l'âme intellective. Tout se passe comme si le démon pensait et la sorcière agissait. Soucieux de souligner l'entière responsabilité de la

${ }^{64}$ Le Marteau des sorcières, Deuxième partie, Première quest. principale, chap. II, éd. cit., p. 253-254; MM, p. 160-161.

${ }_{65}$ Ibid., p. 259-260; MM, p. 167. 
sorcière, les deux inquisiteurs ne font pas de leur ennemie un corps sans âme. Ils se contentent de suggérer, par le biais de leur description, que le démon est devenu la puissance spirituelle qui informe le corps de la sorcière. Pour reprendre les termes de saint Thomas, ce dernier est "proportionné » et «vivifié » par la substance entièrement spirituelle qu'est le démon ${ }^{66}$. Dans le même chapitre dont nous avons tiré ces deux extraits, Henry Institoris et Jacques Sprenger décrivent avec un luxe de détails les terribles onguents dont les sorcières couvrent leur corps : «nous tuons [les enfants] dans leur berceau, même quand ils dorment à côté de leurs parents. Ceux-ci après cela pensent les avoir étouffés ou qu'ils sont morts autrement (de mort naturelle); puis nous les enlevons secrètement de leur tombeau, nous les mettons à cuire dans un chaudron jusqu'à ce que toute la chair se détache des os et devienne bien liquide. De l'élément le plus solide nous faisons un onguent qui nous sert pour nos artifices, nos plaisirs et nos transports. $\rangle^{67}$ Une telle description - qui emprunte son aspect macabre au folklore populaire ${ }^{68}$ - vient nourrir le fantasme des deux inquisiteurs : la sorcière est un corps recouvert de chairs mortes. Le corps sorcier semble être double : à une chair vivante, se superpose un onguent fait de chairs décomposées $^{69}$. Affirmant elle-même que cette étrange préparation lui sert dans les «transports» sabbatiques, la sorcière inventée par les deux auteurs du Malleus appelle de ses vœux une animation démoniaque. Un nouveau composé hylémorphique semble se dresser devant les yeux stupéfaits du lecteur : l'alliance du démon et de la sorcière rappelle étrangement celle de l'âme et du corps.

La description des onguents macabres se clôt par cette précision extrêmement intéressante : "Avec l'élément plus liquide, nous remplissons un récipient comme une outre: celui qui en boira en s'accompagnant de quelques autres cérémonies acquiert immédiatement toute connaissance et devient maître de notre secte. $\gg^{70}$ De la chair décomposée des enfants assassinés, s'élèvent des images très instructives : l'imagination de la sorcière a partie liée avec le corporel le plus dégradé. Encore

\footnotetext{
${ }^{66}$ De malo, question XVI, article 9, éd. cit., p. 859 : proportionatum ; vivificatum.

${ }^{67}$ Le Marteau des sorcières, Deuxième partie, Première quest. principale, chap. II, éd. cit., p. 256 ; hos in cunabulis, vel ad latera iacentes parentum, caremoniis nostris occidimus: quos postquam putantur oppressi esse, vel aliunde mortui, et de tumulo clam furto recipimus, et in caldari decoquimus, quousque evulsis ossibus tota caro efficitur bene potabilis, de solidiore materia unguentum facimus, nostris voluntatibus et artibus, ac transvectionibus accommodis (MM, p. 163).

${ }^{68}$ Voir, à ce propos, Charles Zika, «Les parties du corps, Saturne et le cannibalisme : représentations visuelles des assemblées des sorcières au XVIe siècle», dans Le sabbat des sorciers, éd. cit., p. 389-418.

${ }^{69}$ «Dans un texte où se lit, en continuel filigrane, la haine du corps et de la chair, dans une pensée théologique tournée vers le spirituel, la sorcière vient réintroduire ce qui est le plus concret du corps, le plus charnel» (Nicole Jacques-Chaquin, «Le maléfice de taciturnité. Esquisse d'une étude du mythe de la sorcière», dans Les Cahiers de Fontenay, n ${ }^{\circ}$ 9-10, p. 142). Voir également Yves Pélicier, «Le corps de la sorcière», dans Le Corps à la Renaissance, J. Céard, M. M. Fontaine et J.-C. Margolin éd., Paris, Aux Amateurs de Livres, 1990, p. $139-145$.

${ }^{70}$ Le Marteau des sorcières, Deuxième partie, Première quest. principale, chap. II, éd. cit., p. 256 ; de liquidiore vero humore flasconem ut utrem replemus : de quo is qui potatus fuerit, additis paucis caremoniis, statim conscius efficitur et magister nostra secta (MM, p. 163).
} 
faut-il comprendre ce que les deux auteurs du Malleus empruntent au De malo, et comment la stigmatisation de l'imagination de la sorcière s'enracine encore une fois dans une lecture particulièrement déviante de la philosophie aristotélico-thomiste.

Le démon thomiste, manipulateur d'images : du De malo au Malleus Saint Thomas, dans l'article 1 de la question XVI du De malo, s'inscrit en faux contre la croyance en une quelconque corporéité démoniaque. Le démon néoplatonicien, muni d'un corps, serait capable de véhiculer des images dans l'esprit de sa victime, comme le souligne Thomas d'Aquin lui-même : «saint Augustin dit dans le même livre (XII, 23) ${ }^{71}$ que «si quelque esprit s'empare et se saisit d'une âme, celle-ci est emportée vers la vision d'images corporelles. » Or l'âme ne pourrait pas voir les images des corps dans une substance tout à fait spirituelle. Donc l'esprit de l'ange ou du démon qui s'empare de l'âme a certains organes corporels dans lesquels ces images sont conservées. $»^{72}$ Les auteurs influencés par le platonisme attribuent l'imagination aux créatures démoniaques: «Denys dit dans les Noms Divins $(\mathrm{IV}, 23)$ que le mal chez les démons est «une fureur déraisonnable, un désir insensé et une imagination perverse». Or ces trois éléments appartiennent à la partie sensitive de l'âme, en laquelle se trouvent l'imagination, l'irascible et le concupiscible ; or la partie sensitive n'existe pas sans corps. Donc les démons possèdent des corps qui leur sont naturellement unis. ${ }^{73}$ Dans l'univers néoplatonicien, sa corporéité fait du démon une créature capable d'apparaître sous différentes formes aux yeux de sa victime, ou de conduire de nouvelles images, troublantes et mensongères, dans l'esprit des hommes. Le démon néoplatonicien corporel peut choisir de prendre la forme qu'il désire et trouble ainsi directement l'être humain ${ }^{74}$, s'insinuant dans l'âme de sa victime ou apparaissant concrètement face à elle. Saint Thomas récuse énergiquement de telles affirmations. Privé de corps, le démon thomiste ne véhicule aucune image et ne peut guère apparaître aux yeux des hommes : "l'imagination, qui reçoit son nom de la vision, comme on le dit dans le livre de l'Ame (II, 30), est attribuée métaphoriquement aux démons, comme aussi la vue est attribuée à

${ }^{71}$ Commentaire littéral de la Genèse.

${ }^{72}$ De malo, question XVI, article 1, éd. cit., p. 736 : Augustinus dicit in eodem libro quod «assumente atque rapiente aliquo spiritu tollitur anima ad videndas similitudines corporum ». Non autem posset anima similitudines corporum videre in substantia omnino spirituali. Ergo spiritus angeli vel demonis rapiens animam habet aliqua corporea organa in quibus huiusmodi species conservantur.

${ }^{73}$ Ibid., p. 732 : Dionisius dicit IV cap. De divinis nominibus quod malum in demonibus est «furor irrationabilis, demens concupiscentia et fantasia proterva». Set hec tria pertinent ad partem anime sensitivam in qua est fantasia, irascibilis et concupiscibilis; pars autem sensitiva non est sine corpore. Ergo demones habent corpora naturaliter sibi unita.

${ }^{74}$ Dans le De abstinentia, le néoplatonicien Porphyre écrit par exemple : «Ces derniers [les démons], comme ceux qui ont la propriété contraire, sont tous invisibles et absolument imperceptibles aux sens humains. En effet, ils ne sont pas revêtus d'un corps solide et n'ont pas tous une seule et même figure, mais ils existent sous de nombreux aspects, et leurs figures, qui s'impriment dans leur élément pneumatique et lui donnent sa marque distinctive, tantôt font leur apparition, tantôt restent inapparentes. Parfois même ils changent de figure, les mauvais du moins » (livre II, 39, texte établi et trad. par J. Bouffartigue et M. Patillon, Paris, Belles Lettres, 1979, tome II, p. 105). 
l'intelligence ${ }^{75}$ »; «L'âme a, naturellement unis à elle, les organes corporels qui sont exigés pour ses opérations naturelles ; or apparaitre aux hommes n'est pas une opération naturelle aux démons, ni aucune autre opération pour laquelle est requis un organe corporel ; aussi n'est-il pas nécessaire que les démons aient des corps qui leur soient unis naturellement ${ }^{76}$ »; «comme le démon n'a pas de corps, il ne peut être l'objet des sens, ni même leur moyen ${ }^{77}$. Il semble donc qu'en aucun cas il ne puisse mouvoir les sens ${ }^{78}$ » « «es démons ne peuvent imprimer une forme nouvelle dans les organes corporels des sens $\mathrm{s}^{79} »$.

Le démon thomiste semble encore une fois beaucoup moins puissant que le démon néoplatonicien. Sa faible capacité d'action ne l'empêche pourtant pas de manipuler l'imagination de ses victimes. En d'autres termes, incapable de conduire dans l'âme de l'être humain de nouvelles images, le démon thomiste peut toutefois libérer les images conservées dans la mémoire humaine. Dans l'article 11 de la question XVI du De malo, intitulé «Les démons peuvent-ils changer la partie connaissante de l'âme dans son pouvoir sensitif intérieur ou extérieur? " ${ }^{80}$, saint Thomas met au jour avec beaucoup de précision le mécanisme qui permet au démon de manipuler les images déjà contenues dans l'esprit de l'homme: «le démon excite les sens et l'imagination non certes en imprimant de nouvelles espèces, mais en ramenant vers les organes de l'imagination ou des sens les espèces qui préexistent dans les esprits sensitifs. ${ }^{81}$ Le démon peut produire devant les yeux de sa victime, endormie ou éveillée, une image conservée dans son esprit même. La manipulation démoniaque ne fait appel à aucun surnaturel ; tout se passe comme si le démon thomiste faisait volontairement ce que l'esprit humain fait parfois involontairement :

les démons peuvent, par leur puissance propre, changer localement les corps ; or par le changement local des esprits et des humeurs, il arrive, même naturellement, qu'on voie certaines choses par l'imagination ou les sens. En effet, le Philosophe dit dans le Sommeil et la Veille (3), en assignant la cause des apparitions dans les songes, que lorsqu'un animal s'est endormi, le sang étant descendu en abondance vers le principe des sens, en même temps descendent les mouvements ou les impressions laissées par les mouvements des objets sensibles qui sont conservés dans les esprits

\footnotetext{
${ }^{75}$ De malo, question XVI, article 1, éd. cit., p. 743 : fantasia, que a visione nomen accepit ut dicitur in libro De anima, metaphorice attribuitur demonibus, sicut et visus intellectui.

${ }_{76}$ Ibid., p. 744-745 : anima habet naturaliter sibi unita corporea organa que exiguntur ad naturales eius operationes; apparere autem hominibus non est naturalis operatio demonis, neque aliqua alia ad quam requiratur corporeum organum; unde non oportet quod demones habeant corpora naturaliter sibi unita.

${ }^{77}$ Nous préférons le terme «moyen » à celui de «milieu», utilisé par A. Danet pour traduire medium.

${ }^{78}$ Ibid., article 11, p 862 : demon cum sit incorporeus non potest esse obiectum sensus neque etiam medium. Ergo videtur quod nullo modo possit movere sensum.

${ }^{79}$ Ibid., p. 867 : demones non possunt novam formam imprimere in organa corporea sensuum.

${ }^{80}$ Ibid., p. 860 : Undecimo queritur utrum demones possint immutare partem anime cognoscitivam quantum ad vim sensitivam interiorem vel exteriorem.

${ }^{81}$ Ibid., p. 861 : demon movet sensum et ymaginationem non quidem imprimendo novas species, set species preexistentes in spiritibus sensitivis reducendo ad organum ymaginationis vel sensus.
} 
sensitifs, et ils mettent en mouvement le principe de la connaissance, en sorte que certaines choses apparaissent comme si le principe sensitif était modifié par ces choses extérieures. Et de cette façon, les démons peuvent changer l'imagination et les sens, non seulement de ceux qui dorment, mais même de ceux qui veillent ${ }^{82}$.

Suivant une affirmation aristotélicienne (De l'âme, 431a), saint Thomas souligne que l'âme humaine ne peut jamais penser sans images : "en l'état de la vie présente, nous ne comprenons pas sans images. ${ }^{83}$ La manipulation démoniaque de l'imagination humaine peut donc se révéler extrêmement dangereuse : «il faut donc dire que les démons peuvent, par la puissance de leur nature, en troublant les phantasmes, empêcher complètement la connaissance intellectuelle de l'homme ${ }^{84}$. Comme l'explique Alain Boureau: «Le diable peut agir non sur l'âme, ni sur les puissances opératives, mais sur ces intermédiaires noétiques que sont les species; le démon brouille le message et donc affecte l'intellect qui, tout en restant valide, traite des données corrompues. Dès lors, la volonté, tout en demeurant bonne, subit, dans sa délibération, ce parasitage satanique, un peu comme un virus informatique affecte le fonctionnement d'un logiciel valide. » ${ }^{85}$

Le Malleus retient encore une fois l'essentiel de la démonstration du De malo :

Les apparitions qui arrivent en songes aux dormeurs proviennent du mouvement des formes retenues dans le conservatoire, sous l'effet du mouvement naturel local causé par le flux du sang et des humeurs sur ces puissances sensitives internes, un mouvement intérieur à la tête et aux cellules de la tête. Or cela peut provenir d'un semblable mouvement déclenché par les démons. Et pas simplement chez les dormeurs mais aussi chez les veilleurs. Les démons peuvent remuer les esprits et les humeurs intérieures, pour que les espèces conservées sortent des cachettes vers les principes sensitifs, c'est-à-dire les puissances d'imagination et de fantaisie : ainsi on s'imagine que telle chose existe et c'est cela la tentation intérieure ${ }^{86}$.

\footnotetext{
${ }^{82}$ Ibid., p. 866 : demones virtute propria possunt localiter corpora mutare; ex transmutatione autem locali spirituum et humorum etiam secundum nature operationem contingit aliqua secundum ymaginationem vel sensum videri. Dicit enim Philosophus in libro De sompno et vigilia, assignans causam apparitionis sompniorum, quod cum animal dormierit, descendente plurimo sanguine ad principium sensitivum simul descendunt motus sive impressiones relicte ex sensibilium motionibus que in spiritibus sensibilibus conservantur, et movent principium apprehensivum, ita quod aliqua apparent ac si tunc principium sensitivum a rebus istis exterioribus immutaretur. Et per hunc modum demones possunt immutare ymaginationem et sensum, non solum dormientium set etiam vigilantium.

${ }^{83}$ Ibid., p. 865 : in statu presentis vite non intellegimus absque fantasmate.

${ }^{84}$ Ibid., p. 869-870 : dicendum est ergo quod demones possunt virtute sue nature fantasmata perturbando totaliter intelligibilem cognitionem hominis impedire.

${ }^{85}$ A. Boureau, «Le sabbat et la question scolastique de la personne», art. cit., p. 37.

${ }^{86}$ Le Marteau des sorcières, Première partie, question VII, éd. cit., p. 171-172; Hoc quod contingit in dormientibus de apparitionibus somniorum ex spirituum, id est, specierum in conservatoriis repositarum, et hoc ex naturali motu locali propter commotionem sanguinis et humorum, ad principia illa, id est, ad virtutes sensitivas interiores : et dicimus motu locali intrinseco in capite et in cellulis capitis. Hoc etiam potest accidere ex consimili motu locali per Damones procreato. Et non tantum in dormientibus, sed et vigilantibus : in quibus Da-
} 
Les images libérées dans l'esprit de la victime par la puissance démoniaque ne sont jamais «nouvelles »; leur seule nouveauté pourrait naître d'une combinaison inédite des images déjà contemplées. À la suite de saint Thomas, les deux inquisiteurs reprennent l'exemple de la montagne d'or, association de deux images - la montagne et l'or - toutes deux déjà contenues dans l'esprit humain ${ }^{87}$. Cette présentation de la manipulation démoniaque de l'imagination - qui n'a rien de surnaturel permet à l'auteur du De malo et à ceux du Malleus de faire de Dieu le seul créateur de formes. Le diable ou la sorcière qui ferait sortir ex nihilo une forme devant les yeux stupéfaits de sa victime, ou qui métamorphoserait un être en un autre, concurrencerait dangereusement les pouvoirs créateurs de Dieu lui-même : «Peu de démonologues sont enclins à attribuer au diable des pouvoirs autres que ceux dont disposent les créatures. ${ }^{88}$ Les cathares - versés dans le dualisme le plus extrême ont inventé un Contre-Dieu, un Antithéos, aussi puissant que Dieu lui-même et ont été poursuivis pour hérésie. Les démons néoplatoniciens, corporels et très puissants, auraient également un pouvoir poiétique suspect aux yeux de saint Thomas. En outre, l'anthropologie néoplatonicienne n'interdit pas l'entrée d'une âme humaine dans un corps animal par exemple ${ }^{89}$. Les démons thomistes, dont la capacité est beaucoup plus réduite que leurs homologues néoplatoniciens, «ne peuvent faire que les traits du corps humain se transforment en ceux d'une bête en toute vérité, parce que cela va contre l'ordre mis par Dieu dans la nature ${ }^{90} »$. Les deux inquisiteurs s'inscrivent dans un même cadre de pensée : Dieu «on le sait, peut seul dans sa puissance infinie créer à partir de rien »; il est le seul «créateur de ces essences ${ }^{91}$ ». Les métamorphoses d'hommes en animaux ne sont donc pas réelles mais imaginaires. Le démon, grand manipulateur, libère une image de bête qui restait conservée dans la mémoire de la victime. L'être humain prendra l'homme ou la femme qu'il aperçoit pour un animal: «des hommes donnent l'impression d'être des animaux,

mones possunt admovere \& commovere interiores spiritus et humores, ut species conservatce in conservatoriis educantur de thesauris ad principia sensitiva, id est, ad virtutes illas Imaginativam et Phantasticam, ut res aliquas habeat talis imaginarie: et talis dicetur interior tentatio (MM, p. 76). Voir également Le Marteau des sorcières, éd. cit., p. 185-186, p. 191, p. 193 , p. 285-286, p. 294.

${ }^{87}$ Voir De malo, question XVI, article 11, éd. cit., p. 869 et Le Marteau des sorcières, Première partie, quest. 10, éd. cit., p. 195.

${ }^{88}$ Jean Céard, "Le diable singe de Dieu selon les démonologues des XVI ${ }^{\mathrm{e}}$ et XVII ${ }^{\mathrm{e}}$ siècles », dans Le Diable. Colloque de Cerisy, Cahiers de l'Hermétisme, Paris, éd. Dervy, 1998, p. 32.

${ }^{89}$ Voir, à ce sujet, les analyses de Jean Céard, art. cit., p. 32-33. Voir également J. Céard, «Médecine et démonologie : les enjeux d'un débat», dans Diable, Diables et Diableries au temps de la Renaissance, M.-T. Jones-Davies éd., Paris, J. Touzot, 1988, p. 97-112.

${ }^{90}$ Voir De malo, question XVI, article 9, éd. cit., p. 851-852: non tamen hoc quod humani corporis lineamenta in bestialia convertantur secundum rei veritatem, quia hoc est preter ordinem a Deo nature inditum.

${ }^{91}$ Le Marteau des sorcières, Deuxième partie, Première quest. principale, chap. VIII, éd. cit., p. 290-291; soli Deo convenit, ut notum est, qui sua infinita potentia aliquid ex nihilo creare potest (MM, p 201); talium quidditatum creator (MM, p. 202). 
qui en réalité ne le sont pas $^{92} »$; «le diable peut tromper l'imagination des hommes, au point qu'un homme puisse paraître vraiment un animal ${ }^{93} »$.

Le démon néoplatonicien, contre lequel saint Thomas s'élève énergiquement dans le De malo, serait capable de véhiculer directement des images perverses et mensongères dans l'esprit de ses victimes. Les deux inquisiteurs semblent frémir à la pensée des conséquences possibles d'une telle affirmation. Une créature corporelle, puissante et autonome, n'aurait guère besoin des services d'un agent intermédiaire, d'une sorcière ${ }^{94}$. Mais il y a plus : comment savoir si un être humain est bien la victime d'un démon néoplatonicien qui véhicule des images infernales de l'extérieur à l'intérieur de son esprit, ou s'il ne souffre pas tout simplement d'un délire de sa propre imagination? Les deux inquisiteurs se méfient grandement de la fantaisie comme explication des phénomènes supposés occultes : «Certains, en effet, rapporte saint Thomas dans le Commentaire sur le Livre des Sentences, ont tenté de prouver qu'il n'y a pas de maléfice au monde, sauf dans l'imagination des gens qui attribuent au maléfice des effets naturels de caractère occulte. ${ }^{95}$ Les auteurs du Malleus condamnent «ceux qui disent qu'il n'y a pas de sorcellerie dans le monde mais seulement dans l'opinion des gens ${ }^{96}{ }$. Le démon thomiste, incapable d'apporter

${ }^{92}$ Ibid., Première partie, quest. IX, p. 186 ; homines videntur animalia, cum tamen non sint in rerum veritate ( $M M$, p. 93).

${ }^{93}$ Ibid., quest. X, p. 191 ; Diabolus potest phantasiam hominis decipere, ut homo vere animal videatur (MM, p. 98).

${ }^{94} \mathrm{La}$ relation établie par la tradition néoplatonicienne entre le corps démonique et l'imagination humaine pourrait être vécue comme une menace par les deux inquisiteurs, car elle rend caduque la nécessité du corps sorcier. Nombre de textes néoplatoniciens développent en effet l'idée selon laquelle le spiritus fantasticus, voile d'images qui entoure l'âme, corps subtil de la pensée, est fait de la même substance que les astres dont il provient, mais est aussi de la même nature que le corps subtil des démons, qui servent d'intermédiaires entre le divin et l'homme. La genèse de cette notion de spiritus fantasticus a souvent été retracée. Nous renvoyons, sans bien sûr prétendre à l'exhaustivité, aux études suivantes, classées dans l'ordre chronologique : R. C. Kissling «The ochéma-pneuma of the neoplatonists and the De insomniis of Synesius of Cyrene», American Journal of Philolology, XLIII, 1922, p. 319-330 ; Proclus, The elements of Theology, traduit et commenté par E. R. Dodds, Oxford, Clarendon Press, 1933, Appendix II : "The Astral Body in Neoplatonism», p. 313-321 ; G. Verbeke, L'Evolution de la doctrine du pneuma, du stö̈cisme à saint Augustin, Paris, Desclée De Brouwer; Louvain, Editions de 1'Institut supérieur de philosophie, 1945 ; F. Cumont, Lux perpetua, Paris, Librairie orientaliste Paul Geuthner, 1949, p. 4 ; G. Agamben, Stanze. Parole et fantasme dans la culture occidentale, Paris, Payot et Rivages, 1998, p. 150-170. Plusieurs courants de pensée se conjuguent pour donner naissance à ce spiritus fantasticus: l'association du pneuma d'origine astrale et du sperme chez Aristote; la trace de cette croyance dans le corpus hippocratique, et surtout dans les écrits médicaux grecs d'Erasistrate à Galien; le pneuma placé au centre de la cosmologie et de la psychologie stoïcienne ; l'ochéma-pneuma néoplatonicien.

${ }^{95}$ Le Marteau des sorcières, Première partie, quest. I, éd. cit., p. 106 ; Nam quidam iuxta doctrinam sancti Thoma in 4. distinct. 24. ubi tractat de impedimento maleficiali, conati sunt afferere maleficium nihil esse in mundo nisi in opinione hominum qui naturales effectus, quorum essentice sunt occulte, maleficiis imputabant (MM, p. 2).

${ }_{96}$ Ibid., quest. VIII, p. 181 ; illos, qui dicebant, maleficium nihil esse in mundo, sed tantum esse in opinione hominum (MM, p. 87). 
de l'extérieur de nouvelles images dans l'esprit de sa victime, a très souvent besoin du corps de la sorcière comme support. Le démon est un «moteur» capable de faire voler son «mobile» dans les airs ${ }^{97}$ et de le placer face à un malheureux. Il manipule ensuite l'imagination de sa victime, qui prend alors le corps de la sorcière pour ce qu'il n'est pas : en général un animal très dangereux. Une histoire racontée dans la deuxième partie du Malleus illustre parfaitement cette utilisation démoniaque du corps de la sorcière ${ }^{98}$. Dans une ville du diocèse de Strasbourg, un ouvrier fut attaqué soudainement par trois chats très agressifs. Il réussit enfin à les chasser en les frappant violemment. Peu de temps après, il fut arrêté pour avoir attaqué «trois des matrones les plus considérées de cette ville ${ }^{99}$ ». Comprenant qu'il avait été le jouet d'une illusion diabolique, l'ouvrier affirma «avoir frappé des créatures mais pas des femmes ${ }^{100} »$. Il fut relâché, car les juges comprirent que le pauvre homme avait été victime des démons. Les deux inquisiteurs s'attardent longuement à expliquer le mécanisme d'une telle attaque: «Premièrement, [les matrones] furent pressées d'agir par les instances du démon. [...] Deuxièmement, ayant obtenu leur consentement, les démons ont transféré localement leurs corps, avec cette aisance de la puissance spirituelle supérieure à la force corporelle. Troisièmement, ayant été changées en formes de bêtes par quelque sortilège, de la manière que nous avons dite [à savoir la manipulation de l'imagination de la victime], elles eurent à attaquer l'ouvrier. $»^{101}$ Dans un cadre néoplatonicien, une telle agression aurait pu tout à fait s'expliquer autrement: le démon, muni d'un corps, aurait pu prendre l'apparence illusoire de trois chats; véhicule d'images perverses, il aurait également pu s'infiltrer dans l'esprit de l'ouvrier et lui faire croire à l'existence des trois bêtes. Mais une telle relation entre le corps démoniaque et l'imagination de la victime «courtcircuiterait» en quelque sorte le corps sorcier et ne pourrait pas rendre compte des blessures des trois matrones. La pensée aristotélico-thomiste, si soucieuse de respecter l'ordre naturel de l'univers, sert grandement les deux inquisiteurs. Elle permet de justifier l'existence concrète du corps de la sorcière, instrument non seulement utile mais vivement désiré par les démons : «partout où le démon peut nuire sans la sorcière, il le peut aussi avec la sorcière et même plus facilement, parce que Dieu davantage offensé nous punit ainsi davantage ${ }^{102} »$; le mal, «il faut effectivement

\footnotetext{
${ }^{97}$ De malo, question XVI, article 9, éd. cit., p. 859 : moventis ; mobile.

${ }^{98}$ Le Marteau des sorcières, Deuxième partie, Première quest. principale, chap. IX, éd. cit., p. 297-299; MM, p. 208-211.

${ }_{99}$ Ibid., p. 297 ; tres matronas principuas huius civitatis (MM, p. 209).

${ }^{100}$ Ibid., p. 298 ; creaturas me percussisse recolo, non autem mulieres (MM, p. 209).

${ }_{101}$ Ibid., p. 299; Primo, quod ad Damonum instantiam solicitatce super hoc fuerunt [...] Secundo, habito earum consensu, Damones earum corpora localiter transtulerunt, ea facilitate, qua et spiritualis virtus prestantior corporali existit. Tertio, quod prastigiosa apparitione modo quo supra tactum est, in formas illas bestiales conversa ipsum laboratorem invadere habuerunt (MM, p. 211)

${ }^{102}$ Ibid., chap. XI, p. 308 ; in illis, in quibus Damon per se absque Malefica possit ladere, possit etiam cum Maleficatimo ampliori facilitate propter maiorem Divina maiestatis offensionem, ut supra tactum est (MM, p. 221).
} 
l'imputer à la sorcière, car sans elle Dieu ne permettrait jamais au diable de porter pareille blessure et le diable ne tenterait pas d'aller par lui-même jusque-là ${ }^{103}$ ».

"La différence étant minime entre 'imaginer' et 'fantasmer'104» : l'imagination corporelle de la sorcière dans le Malleus

Le Malleus se montre fidèle à la démonologie thomiste: le démon ne peut pénétrer directement dans l'âme de l'homme, chasse gardée de Dieu. Mais il a pouvoir sur les corps et les imaginations des êtres vivants, et manipule avec beaucoup de dextérité la fantaisie humaine pour faire sortir de leurs «cachettes» certaines images qui y sont conservées ${ }^{105}$ : «bien que le démon ne puisse pas immédiatement opérer à l'intérieur de l'intelligence et de la volonté des hommes, cependant, selon tous les docteurs théologiens, traitant de la puissance d'action des démons sur les corps, ils sont capables d'agir sur les puissances du corps ou liées ou corps, soit sens internes, soit sens externes, si Dieu le permet (saint Thomas, Commentaire sur les Sentences II, 8, I, 5). » ${ }^{106}$ Nous avons vu, en traitant du déplacement sabbatique, que la puissance spirituelle du démon semble vivifier le corps de la sorcière. Cette dernière, s'enduisant d'un onguent fait de chairs d'enfants assassinés, semble redoubler sa propre corporéité. Son corps est alourdi par des lambeaux de cadavres et vivifié par la puissance spirituelle démoniaque. Mais la sorcière conserve une volonté très active, qui fait d'elle un être pleinement responsable de ses actes. Or, cette volonté est intimement liée au statut de l'imagination de la sorcière, si particulier dans le Malleus.

La question XVI de la première partie du Malleus établit une distinction très nette entre le sommeil d'un dormeur dont l'âme est «bien disposée » et celui de la sorcière $^{107}$. Les songes du premier peuvent annoncer certains événements futurs, selon les lois physiologiques établies par Aristote dans Du Sommeil et de la veille. Lorsque les mouvements internes et externes sont apaisés, lorsque les vapeurs des humeurs sont calmées, alors les images oniriques se donnent à lire avec une certaine netteté et peuvent annoncer l'avenir. En outre, certains songes reflètent les dispositions corporelles et peuvent être d'une grande utilité pour les médecins : «Par exemple, si quelqu'un rêve d'incendies, c'est un signe qu'en lui prédomine la bile; s'il rêve d'air, de vol au autre chose, c'est un signe de prédominance sanguine; s'il rêve d'eau ou autre liquide, c'est un signe de l'humeur flegmatique; et s'il rêve de terre, c'est un signe de disposition mélancolique. ${ }^{108}$ Ces deux mécanismes physiologi-

${ }^{103}$ Ibid.,, p. 310 ; merito tamen Malefica imputatur : quia sine ea Deus nunquam lasionem inferre Diabolo permitteret, nec Diabolus per se ladere ipsum attentasset (MM, p. 223).

${ }^{104}$ Ibid., chap. VII, p. 285 ; pauca enim differentia est inter imaginari et phantasiari (MM, p. 196).

${ }^{105}$ Ibid., Première partie, quest. VII, p. 171 ; de thesauris (MM, p. 76).

${ }^{106}$ Ibid., p. 173 ; de primo, quod licet in intellectum et voluntatem hominis immediare Damon nequeat operari, tamen secundum omnes Doctores Theologos in 2. sentent. de virtute Damonis in operando in corpus, et in potentias corporis, sive corpori alligatas, sive sint sensus interiores, sive exteriores, permittente Deo agere valent (MM, p. 79).

${ }^{107}$ Ibid., quest. XVI, p. 222 ; anima disposita (MM, p. 131).

${ }^{108}$ Ibid., p. 222 ; si quis somniat de occupationibus igneis, signum est quod in eo pradominatur cholera; si de aëris, ut de volatu et huiusmodi, signum est sanguinis; si aqua vel alterius liquoris aquei, signum est phlegmatis; si de terrenis signum est melancholice (MM, p. 131). 
ques, parfaitement décrits par Aristote et consciencieusement repris par les deux inquisiteurs, supposent tous deux une certaine passivité du sujet endormi. Les images oniriques contemplées par le dormeur ne sont pas dépendantes de sa propre volonté. Bien différent est le sommeil de la sorcière:

De nouveau cependant tout cela est bien léger en comparaison des songes observés par les sorcières pour leurs maléfices. Car quand elles ne désirent pas être transférées corporellement (on l'a dit) mais percevoir seulement en imagination ce qui est perpétré par leurs collègues sorciers, leur pratique c'est de se coucher sur le côté gauche au nom de leur diable et des démons et alors ces choses leur sont révélées en vision imaginative. Et si elles désirent savoir des démons une chose occulte, soit pour elles-mêmes soit pour d'autres, alors elles les apprennent des démons sur la base d'un pacte passé avec eux non pas tacitement mais expressément ${ }^{109}$.

Dans la deuxième partie du Malleus, les deux auteurs affinent encore leur description : «au nom de tous les démons elles se couchaient pour dormir sur le côté gauche. Alors une sorte de vapeur glauque s'échappait de leurs bouches, au travers de laquelle elles pouvaient voir clairement ce qui se passait. ${ }^{110}$ Le sommeil de la sorcière est un sommeil actif. Ses visions oniriques sont le résultat d'une décision mûrement réfléchie, d'un pacte explicite passé avec le démon. La volonté perverse de la sorcière signe son arrêt de mort, car elle fait d'elle une créature parfaitement responsable de ses maléfices. Le démon thomiste est capable de manipuler l'imagination de sa victime : il ouvre volontairement les vannes de la mémoire et libère des images lascives qui viennent tenter le malheureux dormeur. La sorcière, quant à elle, convoque volontairement des images oniriques. La volonté - du démon et de la sorcière - est le signe certain de la perversité commune de ces deux créatures.

À cette description de la vapeur glauque qui s'élève de la sorcière endormie, succède au chapitre suivant la description d'une vapeur noire qui s'élève de la sorcière forniquant avec le démon. Henry Institoris et Jacques Sprenger, à la suite de saint Thomas, affirment la plupart du temps que le démon n'a pas de corps. Ils suggèrent pourtant - une seule fois dans leur œuvre - que ce dernier peut assumer «un

${ }^{109}$ Ibid., p. 223 ; Sed hac iterum levia sunt, per comparationem ad somnia a Maleficis superstitiose observata. Nam si corporaliter, ut super tactum est, nolunt transferre, sed tantum imaginarie cernere, qua a consodalibus Maleficis perpetrantur, reponere se habent ad sinistrum latus in nomine sui Diaboli et omnium Damoniorum. Unde sit, ut eis singula imaginaria visione reprasententur a simili. Si aliqua occulta scire pro se vel aliis hominibus volunt a Damonibus per somnia instruuntur, non per tacita, sed per expressa cum eis pacta inita (MM, p. 132).

${ }^{110}$ Ibid., Deuxième partie, Première quest. principale, chap. III, p. 268 ; illa in nomine omnium Diabolorum ad latus sinistrum se reponeret ad cubitum : ex tunc quasi vapor quidam glaucus ex eius ore procederet. Unde singula qua ibi agerentur perlucide considerarent (MM, p. 176). 
corps aérien, mais qui d'une certaine manière est terrestre ${ }^{111} »$. Le démon a en effet parfois besoin d'un corps pour forniquer avec la sorcière : «Mais pour ce qui est des spectateurs, les sorcières elles-mêmes ont souvent été vues couchées sur le dos dans les champs et les forêts, nues jusqu'au-dessus du nombril ; et en position pour cette turpitude, agitant les jambes et les cuisses, les membres tout prêts ; les démons incubes étant en action, même si c'était invisiblement pour les spectateurs et même parfois à la fin de l'acte une vapeur très noire de la longueur d'un homme s'élevait audessus de la sorcière. ${ }^{112}$ La vapeur noire du corps démoniaque ressemble étrangement à la vapeur glauque des songes de la sorcière ${ }^{113}$. Toutes deux ont rapport à l'imaginaire corporel le plus dégradé. De la bouche de la sorcière s'élèvent des images lascives qui représentent les turpitudes sexuelles et macabres du sabbat; du sexe de la sorcière sort le corps démoniaque qui vient de forniquer avec elle d'une hideuse façon. Rappelons également que les sorcières avouent apprendre tous les secrets de leur science en buvant un jus né de la décomposition de chairs d'enfants assassinés : «on boit de l'outre ci-dessus décrite; et aussitôt on se sent intérieurement concevoir toutes les images de notre art et retenir tous les rites de notre secte. $»^{114}$ Tout comme ils avaient redoublé la corporéité de la sorcière, les deux inquisiteurs rangent également son imagination du côté du corps. La sorcière est-elle encore un être humain comme les autres ? La question mérite d'être posée, malgré les allégations des deux inquisiteurs déjà rencontrées. De l'âme de la sorcière ont disparu toute dimension spirituelle et toute influence divine. Sa chair est alourdie par le poids très concret des corps décomposés de ses victimes. Son imagination ne fait plus le lien entre le corporel et le spirituel, mais est rangée définitivement du côté de la chair la plus dégradée. Seule sa volonté perverse fait de la sorcière un sujet entièrement responsable de ses actes et qui mérite la peine capitale.

Ce statut si particulier d'une imagination «corporelle» de la sorcière mérite d'être comparé au statut aristotélico-thomiste de la fantaisie. Selon le Stagirite, «l'âme ne pense jamais sans images ${ }^{115}$ ». Cette affirmation est à mettre en rapport avec la quête de l'abstraction, engagée par l'âme humaine.

${ }^{111}$ Ibid., chap. IV, p. 269 ; aëreum corpus assumit, et quod aliquo modo est terrestre (MM. p. 178). Voir à ce propos, A. Boureau, «Le sabbat et la question scolastique de la personne», art. cit., p. 36.

${ }^{112}$ Ibid., p. 276-277 ; Tametsi quo ad circunstantes, sapius ipsa Malefica supina iacentes in agris seu sylvis visce sunt, et denudatce supra umbilicum, et iuxta dispositionem illius spurcitia coaptatis membris, tibiis et cruribus se agitantes. Damonibus Incubis invisibiliter ibidem quo ad circunstantes cooperantibus: licet in fine actus, vapor negerrimus in longitudine hominis sursum a Malefica in aëre elevabitur (MM, p. 186-187).

${ }^{113}$ Pour la relation entre les rêves et le diable au Moyen Âge, voir Jacques Le Goff et Nicolas Truong, Une histoire du corps au Moyen Âge, éd. Liana Levi, 2005, «Les rêves sous surveillance », p. 86-96.

${ }^{114}$ Le Marteau des sorcières, Deuxième partie, Première quest. principale, chap. II, éd. cit., p. 256 ; de utre bibit supradicto, quo facto, statim se in interioribus sentit imagines nostrae artis concipere et retinere super principales ritus huius sectoe (MM, p. 164).

${ }^{115}$ Aristote, De l'âme, 431a. Voir, à ce propos, Jean-Louis Labarrière, « Jamais l'âme ne pense sans phantasme », dans Aristote et la notion de nature, textes réunis et présentés par PierreMarie Morel, Bordeaux, Presses universitaires de Bordeaux, 1997, p. 149-179. 
Plus l'âme se dégage de la matière, plus elle s'élève. Plus elle est en acte, moins elle est soumise passivement à la matière et à son indétermination. Plus elle se détermine en tant que liberté active, et plus elle est en acte. Il y a donc, dans l'actualisation de l'âme, plusieurs niveaux. Au plus haut niveau se trouve l'intellect, qui comporte encore deux niveaux : l'intellect passif et l'intellect actif. L'intellect passif serait, pourrait-on dire, celui des captifs enchaînés dans la caverne de Platon : ils ont besoin des ombres pour pouvoir penser, ils dépendent donc encore de l'extérieur, de la matière. L'intellect actif, en revanche, acquiert son autonomie, il s'arrache à toute passivité, à la matière. Il est alors éternel ${ }^{116}$.

L'imagination accompagne l'âme humaine dans sa lente remontée ; toute la question étant de savoir si l'âme peut accéder à l'abstraction la plus complète ${ }^{117}$. Saint Thomas emprunte à Aristote cette fonction accompagnatrice de l'imagination. Christophe Bouriau donne un bon aperçu du statut thomiste de la fantaisie, dans ses annotations du De imaginatione de Jean-François Pic de la Mirandole :

Entre l'imagination et l'intellect, entre le fantasme et l'espèce intelligible, le particulier et l'universel, Thomas pose une différence de genre : «sunt alterius generis» (De anima, qu. 4, ad 5). Cependant l'imagination pourvoyeuse de phantasmes est requise pour que la connaissance intellectuelle soit possible (De Veritate, X, 6, ad 7). En effet, les objets de la connaissance humaine comportent un élément universel et intelligible, associé à un élément particulier et matériel. L'opération propre à l'intellect agent consiste à dissocier ces deux éléments, afin de fournir à l'intellect possible l'intelligible et l'universel qui se trouvaient impliqués dans le sensible. Le propre de l'intellect humain est d'abstraire la forme de la matière individuelle que les phantasmes de l'imagination lui présentent (S. Theol., I, 85, 1, ad resp.). Cette abstraction consiste en ce que l'intellect agent appréhende dans chaque chose matérielle ce qui la constitue dans son espèce, en laissant de côté les principes d'individuation qui appartiennent à sa matière (son lieu et son temps, ses qualités sensibles propres). Il peut ainsi considérer à part, dans les fantasmes que lui livre l'imagination, ce qui constitue l'essence de l'homme, du cheval ou de la pierre, sans tenir compte de ce qui distingue, au sein de ces espèces, tel ou tel individu particulier (ibid, I, 85, 1, ad $1)^{118}$.

Les deux inquisiteurs se souviennent parfaitement de ce statut aristotélicothomiste de l'imagination. Le De malo affirme que «l'âme ne comprend jamais sans images $^{119}$ », « nous ne comprenons pas sans images ${ }^{120} »$. Par suite, le démon, ce mani-

\footnotetext{
${ }^{116}$ J. Hersch, L'étonnement philosophique, éd. cit., p. 65.

${ }^{117}$ Les études sur la conception aristotélicienne de l'imagination sont très nombreuses. Nous renvoyons à l'ample bibliographie établie par Pierre-Marie Morel dans Aristote, Petits traités d'histoire naturelle, traduction et présentation par P.-M. Morel, Paris, Flammarion, 2000, p. 219-226.

${ }^{118}$ Jean-François Pic de la Mirandole, De l'imagination. De imaginatione, édité par Christophe Bouriau, éd. comp'Act, 2005, p. 104. Voir également Robert Edward Brennan, «The Thomistic Concept of Imagination », dans The New Scholasticism, 15, 1941, p. 149-161.

${ }^{119}$ De malo, question XVI, article 8, éd. cit., p. 836 : nequaquam sine fantasmate intelligit anima.
} 
pulateur de la fantaisie, «remplit de certaines brumes toutes les avenues de l'intelligence, par lesquelles le rayonnement de la pensée fait passer habituellement la lumière de la raison ${ }^{121} »$. Les deux auteurs du Malleus sont entièrement tributaires de la pensée de saint Thomas: "En conséquence, puisque toute notre connaissance part du sens - selon le Philosophe, les fantasmes sont nécessaires à la spéculation le démon qui vient perturber la fantaisie interne peut donc aussi enténébrer l'intelligence. Ce qui n'est pas agir directement sur l'âme, mais par la médiation des fantasmes. ${ }^{122}$ La sorcière convoque, par le biais de sa volonté, les images fortement sexualisées du sabbat. La victime des démons voit soudainement apparaître en son esprit une image lascive. La fantaisie n'accompagne plus la belle remontée de l'âme vers l'abstraction ou vers le monde divin, bien au contraire... L'image érotique et dangereuse, sortie des cachettes de l'esprit humain où elle sommeillait, se donne à voir, par l'entremise des démons, comme une nouvelle perception. L'imagination ne fait plus le lien entre l'âme et le corps ; elle force l'homme à rester le jouet de la matérialité.

Dans un article intitulé «Le diable en procès », Martine Ostorero et Étienne Anheim posent une question très délicate : «Serait-ce la démonologie thomiste qui aurait ouvert la voie aux chasses aux sorcières ? ${ }^{123}$ Au terme de cette étude, il semble bien que les deux auteurs du Malleus, «manuel de base des juges chargés de poursuivre et d'éliminer la sorcellerie, spécifiquement la sorcellerie féminine ${ }^{124}$ ", suivent scrupuleusement la démonologie développée dans la question XVI du De malo. Tout se passe comme si les deux inquisiteurs avaient donné corps à toutes les indications, parfois relativement sommaires, de cet ouvrage. Mais cette influence est d'autant plus ironique que saint Thomas, très certainement animé par un «christianisme de la lumière », pour reprendre une belle expression d'Alain Boureau $^{125}$, avait voulu limiter le plus possible la puissance des démons. Tenant compte du désintérêt aristotélicien pour ces créatures intermédiaires, il a tenté de limiter leur champ d'action. Privés de corps, les démons thomistes ne sont guère puissants. Incapables de véhiculer des images nouvelles dans l'esprit des hommes, ils doivent se contenter de manipuler la fantaisie de leur victime. Or ces deux «limitations» offrent justement aux deux inquisiteurs - et à l'Église catholique - un cadre rêvé pour justifier la nécessité du corps sorcier. La sorcière devient un médium indispensable aux démons, qui ne pourraient guère agir sans elle dans le monde sensible.

\footnotetext{
${ }^{120}$ Ibid., article 11, p. 865 : non intelligimus absque fantasmate.

${ }^{121}$ Ibid., article 12, p. 875 : quibusdam nebulis implet omnes meatus intelligentie per quos pandere lumen rationis radius mentis solet.

${ }^{122}$ Le Marteau des sorcières, Première partie, quest. VII, éd. cit., p. 174 ; Consequenter cum omnis nostra cognitio intellectiva ortum habet a sensu (cum intelligentem, iuxta Philosophum in 2. de anima, necesse sit phantasmata speculari), ideo sicut Damon potest interiorem phantasiam immutare, sic etiam intellectum obtenebrare. Et hoc quidem non erit immediate agere in animam, sed mediantibus phantasmatibus (MM, p. 79).

${ }^{123}$ Martine Ostorero, Étienne Anheim, «Le diable en procès », dans Médiévales, $n^{\circ} 44$, Paris, PUV, printemps 2003, p. 6.

${ }^{124}$ Le Marteau des sorcières, introduction, éd. cit., p. 5.

${ }^{125}$ A. Boureau, Satan hérétique, éd. cit., p. 141.
} 
«Mobile» mu par un «moteur», la sorcière offre son corps comme support aux illusions démoniaques.

La sorcière fabriquée de toutes pièces dans le Malleus est la conséquence terrible «d'un thomisme un peu étriqué quand il (n'est) plus illuminé par une lumière humaniste ${ }^{126} »$. Toute déviance, toute perversion de la pensée implique un usage des mêmes concepts, mais à des fins négatives. L'âme humaine aspire à son corps selon saint Thomas : elle serait, sans lui, «condamnée à la stérilité et à l'inaction, à moins qu'elle ne se donne un instrument, incomplet aussi sans elle, qu'elle organisera, animera du dedans ${ }^{127}$ ». Il est tentant de lire ce passage à la manière des deux inquisiteurs, dignes représentants de l'Église du $\mathrm{XV}^{\mathrm{e}}$ siècle finissant: le démon, privé de corps, serait condamné à la stérilité et à l'inaction, à moins qu'il ne se donne un instrument - la sorcière - incomplet aussi sans lui, qu'il organisera, animera du dedans. Pour parvenir à ses fins, l'institution catholique se comporte comme de nombreux bourreaux par rapport à leurs victimes: elle tente de se débarrasser de l'âme de la sorcière et assimile l'imagination et le fantasme... La perversion est aussi ancienne que l'esprit humain : le Malleus en est un exemple frappant.

Christine Pigné

Université de Rennes 2 - CELAM

${ }^{126} I d$., « Le sabbat et la question scolastique de la personne», art. cit., p. 44.

${ }^{127}$ É. Gilson, Le Thomisme, éd. cit., p. 245-246. 